\title{
CRESCIMENTO E ACÚMULO DE NITROGÊNIO E POTÁSSIO PELO MELÃo PELE DE SAPO FERTIRRIGADO
}

\author{
Amansleone da S. Temóteo ${ }^{1}$; José Francismar de Medeiros; Indalécio Dutra; Francisco \\ de Assis de Oliveira \\ ${ }^{I}$ Departamento de Engenharia Rural, Faculdade de Ciências Agronômicas, Universidade Estadual Paulista, \\ Botucatu, SP, amanst@fca.unesp.br
}

\section{RESUMO}

Com o objetivo de obter curvas de massa seca e de acúmulo de nitrogênio e potássio pelo melão Pele-de-Sapo (Cucumis melo L.) híbrido Sancho, sob fertirrigação e diferentes lâminas de irrigação, conduziu-se um experimento em Mossoró-RN, num delineamento experimental em blocos casualizados com três repetições. Os tratamentos consistiram de doses, em kg ha ${ }^{-1}$, de nitrogênio: 0, 91, 140 e 184 e de potássio: 0, 174, 260 e 346 aplicadas via fertirrigação sob três lâminas de irrigação: 281, 349 e $423 \mathrm{~mm}$. Realizou-se cinco coletas da parte aérea das plantas (caule, folha e fruto) a partir dos 28 até 70 dias após transplantio, em intervalos de 10 dias, para determinações da massa seca da parte aérea e acúmulo de $\mathrm{N}$ e $\mathrm{K}$. Doses superiores a 140 e $260 \mathrm{~kg} \mathrm{ha}^{-1}$ de $\mathrm{N}$ e K (utilizadas pelos produtores da região) respectivamente não promoveram aumento no acúmulo de fitomassa seca total. As doses crescentes de nitrogênio garantiram os maiores acúmulos deste nutriente ao longo do ciclo da planta e as doses de $\mathrm{N}$ de 91 e de $140 \mathrm{~kg} \mathrm{ha}^{-1}$, e de $\mathrm{K}$ de 174 e de $260 \mathrm{~kg} \mathrm{ha}^{-1}$ e a lâmina de $349 \mathrm{~mm}$ proporcionaram maior acúmulo de potássio na planta.

UNITERMOS: Cucumis melo L., nutrição mineral, lâminas de irrigação.

\section{TEMÓTEO, A. da S.; MEDEIROS, J. F. de; DUTRA, I. OLIVEIRA, F. A. de; GROWTH AND ABSORPTION OF N AND K BY FERTIGATED FROG SKIN MELON}

\section{ABSTRACT}

With the objective of obtaining dry matter curves, and nitrogen and potassium accumulation in Piel de Sapo melon (Cucumis melo L.) hybrid Sancho, an experiment was conducted in Mossoró - $\mathrm{RN}$ in a randomized blocks design with three repetitions. Treatments consisted of doses in kg ha-1 of nitrogen: 0, 91, 140 and 184 and potassium: 0, 174, 260 and 346 ; which were applied by fertigation in depths of 281,349 and $423 \mathrm{~mm}$. Stems, leaves and fruits were harvested 5 times at 28 to 70 days after transplantation, in intervals of 10 days for analyses of aerial dry matter, nitrogen, and potassium accumulation. Doses higher than 140 and $260 \mathrm{~kg}$ ha-1 of $\mathrm{N}$ and $\mathrm{K}$ respectively, did not induce greater accumulation of these nutrients increasing levels throughout the plant cycle. Nitrogen doses of 91 and $140 \mathrm{~kg}$ ha-1, potassium doses of 174 and $260 \mathrm{~kg}$ ha-1 as well as depth of $348 \mathrm{~mm}$ provided higher accumulation of potassium in the plant.

KEY WORDS: Cucumis melo L., mineral nutrition, irrigation depths. 


\section{INTRODUÇÃO}

A quantidade e a proporcionalidade dos nutrientes absorvidos pelas plantas são funções de características intrínsecas do vegetal, como, também dos fatores externos que condicionam o processo. Assim, as informações das exigências nutricionais são fundamentais para assegurar a máxima eficácia e utilização dos fertilizantes sem provocar excesso, conseguindo um desenvolvimento ótimo para cultura (Vivancos, 1996).

A análise de crescimento permite avaliar o crescimento final da planta como um todo e a contribuição dos diferentes órgãos para o crescimento total, podendo assim estimar as causas de variações de crescimento entre plantas semelhantes em ambientes diferentes (Benincasa, 2003). Em relação ao meloeiro, estudos da análise de crescimento sob diferentes condições ambientais demonstram curvas de crescimento com três diferentes estágios onde, o primeiro apresenta taxa de acúmulo lenta, o segundo com rápido crescimento e o terceiro o acúmulo decresce em relação ao segundo.

As plantas possuem em média, cerca de $5 \%$ de nutrientes minerais na massa de material seco, existindo assim grandes diferenças entre espécies, quanto às quantidades exigidas. A absorção de nutrientes difere de acordo com a fase de desenvolvimento da planta, intensificando-se com o florescimento, a formação e crescimento dos frutos. A curva de acúmulo de nutrientes de diversas hortaliças utilizada como parâmetro para recomendação de adubação, é uma boa indicação da necessidade de nutrientes em cada etapa de desenvolvimento da planta.

Os macronutrientes nitrogênio e potássio são encontrados em maiores quantidades pela cultura, exercendo grande influência na produção e na qualidade dos frutos do meloeiro. Com uso da fertirrigação, torna-se fácil à adaptação das quantidades e concentrações dos nutrientes específicos exigidos pelas culturas em cada fase de desenvolvimento. Como conseqüência, a lixiviação de nutrientes para fora do bulbo úmido é reduzida (Bar-Yoseff, 1999). Na ausência de uma curva de absorção de nutrientes, a produção de massa seca fornece uma boa aproximação do acúmulo de nutrientes (Souza \& Coelho, 2001), fundamental para se planejar o parcelamento das doses dos nutrientes a serem aplicadas. Nesse contexto, o trabalho teve como objetivo obter curvas de crescimento de material seco e de acúmulo de nitrogênio e potássio no melão pele de sapo em diferentes fases de desenvolvimento da cultura.

\section{MATERIAL E MÉTODOS}

A pesquisa foi realizada no período de setembro a dezembro de 2004, na fazenda Norfruit Ltda, no município de Mossoró, RN. O solo da área foi classificado como Argissolo Vermelho-Amarelo Eutrófico latossólico (EMBRAPA, 1999) com as seguintes características: $\mathrm{Ca}=2,75, \mathrm{Mg}=1,35, \mathrm{~K}=0,47, \mathrm{Na}=0,041, \mathrm{Al}=0,0\left(\mathrm{em} \mathrm{cmol}_{\mathrm{c}} \mathrm{dm}^{-3}\right)$ e $\mathrm{P}=$ $1,92 \mathrm{mg} \mathrm{dm}^{-3}, \mathrm{pH}=7,6$. A água de irrigação foi proveniente de poço que explora o aqǘfero calcário Jandaíra, que apresentam as seguintes características: $\mathrm{CE}\left(\mathrm{dS} \mathrm{m} \mathrm{m}^{-1}\right)=2,7, \mathrm{pH}=7,0, \mathrm{Ca}$ $=9,0, \mathrm{Mg}=4,7, \mathrm{~K}=0,12, \mathrm{Na}=14,8, \mathrm{Cl}=16,8, \mathrm{HCO}=7,0, \mathrm{CO}_{3}=0,4\left(\mathrm{em} \mathrm{mmol}_{\mathrm{C}} \mathrm{L}^{-1}\right)$, EMBRAPA (1997).

O preparo do solo antes do plantio foi de forma idêntica ao empregado pelo produtor, nos plantios comerciais, constando de uma aração, gradagem para destorroamento, e 
confecção de camalhões de plantio com grade enleiradeira na faixa de solo, e em seguida foi feita a cobertura dos camalhões com plástico (mulch).

O controle fitossanitário e as capinas também foram realizados de acordo com o manejo adotado pela fazenda. Inseticidas específicos foram aplicados por ocasião do surgimento de pragas, sobretudo da mosca branca (Bemisia argentifolii) e minadora (Liriomyza sp.). Foram realizadas capinas manuais, principalmente nas entrelinhas, visto que a cobertura do solo auxilia no controle preventivo de ervas daninhas.

Os tratamentos consistiram de doses, em $\mathrm{kg} \mathrm{ha}^{-1}$, de $\mathrm{N}$ e $\mathrm{K}$, que variaram de 0 a 3 , aplicadas via fertirrigação sob lâminas de irrigação distintas, L1, L2 e L3 a saber: N0: 0; N1: 91; N2: 140 e N3: 184; K0: 0; K1: 174; K2: 260 e K3: 346 e, L1: 281; L2: 349 e L3: 423 mm, sendo que, para cada lâmina de irrigação foram testadas as doses de N1K1, N2K2, N3K3, N0K2 e N2K0.

O delineamento usado foi o de blocos inteiramente casualizados com três repetições, totalizando 15 parcelas por lâminas de irrigação, constituídas de uma fileira de $6,4 \mathrm{~m}$ de comprimento contendo 16 plantas.

A adubação de base correspondeu a $39 \mathrm{~kg} \mathrm{ha}^{-1}$ de $\mathrm{N}$ e $296 \mathrm{~kg} \mathrm{ha}^{-1}$ de $\mathrm{P}_{2} \mathrm{O}_{5}$. O complemento nutricional do fósforo foi realizado via fertirrigação utilizando-se ácido fosfórico, no total de $148,6 \mathrm{~kg} \mathrm{ha}^{-1}$ de $\mathrm{P}_{2} \mathrm{O}_{5}$. O plantio foi realizado 11 dias após o semeio com mudas de melão tipo Pele de Sapo (Cucumis melo L.) híbrido Sancho num espaçamento de $1,85 \mathrm{~m} \times 0,4, \mathrm{~m}$. As plantas foram coletadas aos 28, 38, 49, 58 e 70 dias após transplantio (DAT), sendo amostrada uma planta por parcela previamente sorteada. Os conteúdos foram determinados a partir dos teores de $\mathrm{N}$ e $\mathrm{K}$ e da matéria seca da parte área total da cultura (caule + folha + fruto) segundo Tedesco et al. (1995). As análises de variância e regressão foram realizadas através do SAEG 8.0 (Ribeiro Júnior, 2001).

\section{RESULTADOS E DISCUSSÃO}

As lâminas de irrigação L2 e L3, de 349 e 423 mm, respectivamente, e os tratamentos que continham $\mathrm{N}$ mais $\mathrm{K}$ na composição garantiram aumentos dos acúmulos de fitomassa seca, de nitrogênio e de potássio na parte aérea ao longo do ciclo da cultivar (Figuras 1B, 1D e 1F). Ao final do ciclo não foi observada diferença significativa entre as lâminas de irrigação, no entanto, considerando os resultados em valores absolutos, verifica-se que a lâmina de 349 $\mathrm{mm}$ (L2) e os tratamentos que continham $\mathrm{N}$ mais $\mathrm{K}$ também foram aqueles que garantiram os maiores acúmulos de $\mathrm{N}$ dos 40 aos 70 DAT (Figura 1). Assim como em outros trabalhos de exigência nutricional em cucurbitáceas, também nessa pesquisa o potássio foi o nutriente mais absorvido (Silva Júnior et al., 2006; Oliveira et al., 2009). Para o acúmulo de potássio, a lâmina L2 foi a mais efetiva, no entanto, o uso da dose mais elevada de $\mathrm{N}$ e K (N3K3) não assegurou os mesmos resultados obtidos para o acúmulo de nitrogênio; os maiores acúmulos de potássio ocorreram no período de 40 a 70 DAT. Dessa forma, a lâmina L2 foi a que, possivelmente, proporcionou teores adequados de umidade no solo, promovendo baixa lixiviação e consequentemente maior absorção pela planta, o que poderia justificar os resultados obtidos. 

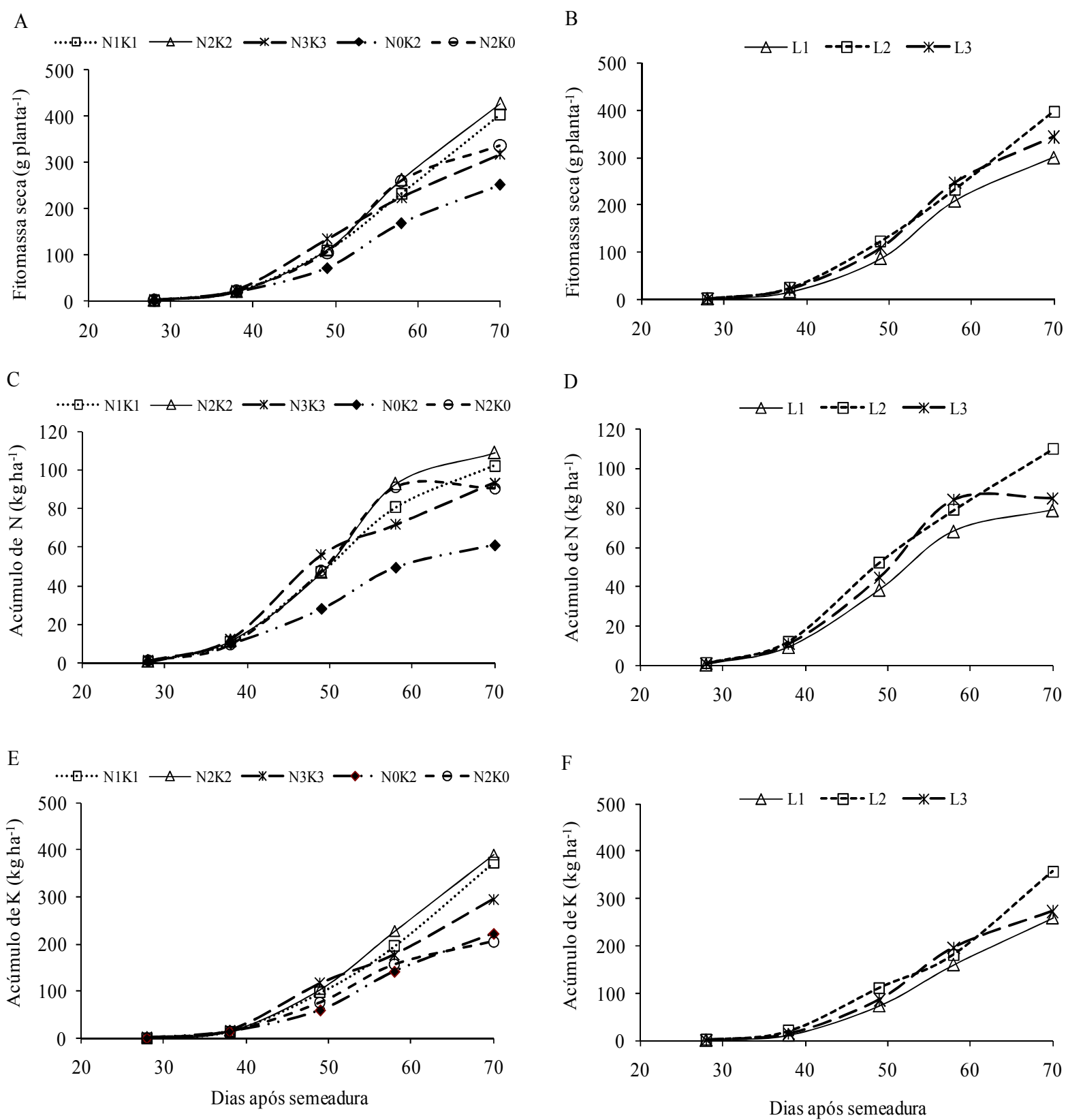

Figura 1. Curvas de massa seca da parte aérea $(A-B)$ e de acúmulo de nitrogênio $(C-D)$ e potássio (E-F) na parte aérea de Cucumis melo L., var. Pele de Sapo, fertirrigado com diferentes doses de nitrogênio, potássio e lâminas de irrigação durante o ciclo.

O período de maior exigência de $\mathrm{N}$ e $\mathrm{K}$ ocorreu entre os 46 e 63 DAT (Tabelas 2 e 3), coincidindo com o período de máximo acúmulo de fitomassa seca, em conseqüência, provavelmente, de neste período, a cultura se encontrar na fase de crescimento e maturação de frutos, sendo os frutos, drenos preferenciais (Oliveira et tal. 2009).

A falta de nitrogênio (N0K2) proporcionou diminuição no acúmulo de fitomassa seca, isso se deu provavelmente pela baixa capacidade fotossintética, proporcionada pela ausência desse nutriente. Independentemente da lâmina aplicada, o crescimento do meloeiro foi lento inicialmente, com incremento na taxa de crescimento no período compreendido entre 38 aos 58 DAT e um declínio ao final do ciclo (Figura 1). Esse comportamento também foi 
observado por Lima (2001), Silva (2002), Farias et al. (2003) e Silva Júnior et al. (2006) em outras pesquisas com meloeiro.

A partir da derivação das equações ajustadas para fitomassa seca e acúmulos de $\mathrm{N}$ e de $\mathrm{K}$, em kg ha ${ }^{-1}$ (Tabelas 1,2 e 3) pode-se obter as taxas diárias e máximas de fitomassa seca e de nutrientes ( $\mathrm{N}$ e $\mathrm{K})$.

Esses resultados demonstram que doses de 91 e $260 \mathrm{~kg} \mathrm{ha}^{-1}$ de $\mathrm{N}$ e $\mathrm{K}$, respectivamente (N2K2), e lâmina de irrigação de $349 \mathrm{mmm}$ (L2) podem ser recomendas para a cultura do meloeiro, var. pele de sapo, visto que, aplicando-se esses níveis de nutrientes e de água obteve-se máximo acúmulo de fitomassa seca, de $\mathrm{N}$ e de $\mathrm{K}$.

Tabela 1. Equações que estimam a fitomassa seca da parte aérea (FSPA), em g planta ${ }^{-1}$, em função dos dias após semeadura (DAS), das doses de $\mathrm{N}$ e $\mathrm{K}$ e diferentes lâminas de irrigação.

\begin{tabular}{|c|c|c|c|c|c|c|}
\hline \multirow{3}{*}{ Modelos de FSPA } & \multirow{2}{*}{\multicolumn{3}{|c|}{ Parâmetros }} & \multicolumn{3}{|c|}{ Idade (DAS) } \\
\hline & & & & \multirow[b]{2}{*}{$\mathrm{R}^{2}$} & Para FSPA & Para taxa de \\
\hline & A & $\mathrm{B}$ & $\mathrm{C}$ & & máximo & Crescimento máximo \\
\hline $\mathrm{N} 1 \mathrm{~K} 1=\mathrm{a} /\left(1+(\mathrm{x} / \mathrm{b})^{\wedge} \mathrm{c}\right)$ & $574,352^{*}$ & $61,441^{*}$ & $-6,557^{*}$ & 0,999 & $70 \mathrm{DAS}$ & $58 \mathrm{DAS}$ \\
\hline $\mathrm{N} 2 \mathrm{~K} 2=\mathrm{a} . \exp \left(-0.5((\mathrm{x}-\mathrm{b}) / \mathrm{c})^{\wedge} 2\right)$ & $43,484^{*}$ & $71,725^{*}$ & $13,83^{*}$ & 0,999 & 70 DAS & $57 \mathrm{DAS}$ \\
\hline $\mathrm{N} 3 \mathrm{~K} 3=\mathrm{a} /\left(1+(\mathrm{x} / \mathrm{b})^{\wedge} \mathrm{c}\right)$ & $368,131^{*}$ & $53,804^{*}$ & $-6,875^{*}$ & 0,998 & $70 \mathrm{DAS}$ & $51 \mathrm{DAS}$ \\
\hline $\mathrm{N} 0 \mathrm{~K} 2=\mathrm{a} /(1+\exp (-(\mathrm{x}-\mathrm{b}) / \mathrm{c}))$ & $275,327^{*}$ & $55,29^{*}$ & $6,224^{*}$ & 0,999 & 70 DAS & 55 DAS \\
\hline $\mathrm{N} 2 \mathrm{~K} 0=\mathrm{a} /(1+\exp (-(\mathrm{x}-\mathrm{b}) / \mathrm{c}))$ & $347,517^{*}$ & $52,838^{*}$ & $4,886^{*}$ & 0,999 & 70 DAS & $51 \mathrm{DAS}$ \\
\hline $\mathrm{L} 1=\mathrm{a} \cdot \exp \left(-0.5((\mathrm{x}-\mathrm{b}) / \mathrm{c})^{\wedge} 2\right)$ & $302,036^{*}$ & $68,882^{*}$ & $12,662^{*}$ & 0,999 & 70 DAS & 56 DAS \\
\hline $\mathrm{L} 2=\mathrm{a} /\left(1+(\mathrm{x} / \mathrm{b})^{\wedge} \mathrm{c}\right)$ & $593,328^{*}$ & $62,176^{*}$ & $-5,909^{*}$ & 0,999 & 70 DAS & 58 DAS \\
\hline $\mathrm{L} 3=\mathrm{a} \cdot \exp \left(-0.5((\mathrm{x}-\mathrm{b}) / \mathrm{c})^{\wedge} 2\right.$ & $345,623^{*}$ & $68,722^{*}$ & $13,029^{*}$ & 0,999 & $70 \mathrm{DAS}$ & $55 \mathrm{DAS}$ \\
\hline
\end{tabular}

(*): significativo (ns): não significativo a $5 \%$ pelo teste $\mathrm{T}$.

Tabela 2. Equações que estimam o acúmulo de nitrogênio na planta (CNPL), em kg ha-1, em função dos dias após semeadura (DAS), para diferentes doses de $\mathrm{N}$ e $\mathrm{K}$ e diferentes lâminas de irrigação.

\begin{tabular}{|c|c|c|c|c|c|c|}
\hline \multirow{3}{*}{ Modelos de CNPL } & \multirow{2}{*}{\multicolumn{3}{|c|}{ Parâmetros }} & \multicolumn{3}{|c|}{ Idade (DAS) } \\
\hline & & & & & Para & \\
\hline & A & b & c & $\mathrm{R}^{2}$ & máximo & máximo \\
\hline $\mathrm{N} 1 \mathrm{~K} 1=\mathrm{a} /\left(1+(\mathrm{x} / \mathrm{b})^{\wedge} \mathrm{c}\right)$ & $111,746^{*}$ & $51,157^{*}$ & $-7,553^{*}$ & 0,999 & $70 \mathrm{DAS}$ & $49 \mathrm{DAS}$ \\
\hline $\mathrm{N} 2 \mathrm{~K} 2=\mathrm{a} /(1+\exp (-(\mathrm{x}-\mathrm{b}) / \mathrm{c}))$ & $112,296^{*}$ & $50,382^{*}$ & $5,182^{*}$ & 0,999 & $70 \mathrm{DAS}$ & $50 \mathrm{DAS}$ \\
\hline $\mathrm{N} 3 \mathrm{~K} 3=\mathrm{a} /\left(1+(\mathrm{x} / \mathrm{b})^{\wedge} \mathrm{c}\right)$ & $97,282^{*}$ & $48,163^{*}$ & $-7,121^{*}$ & 0,999 & $70 \mathrm{DAS}$ & $46 \mathrm{DAS}$ \\
\hline $\mathrm{N} 0 \mathrm{~K} 2=\mathrm{a} . \exp \left(-0.5((\mathrm{xb}) / \mathrm{c})^{\wedge} 2\right)$ & $61,483^{*}$ & $68,276^{*}$ & $15,535^{*}$ & 0,999 & $70 \mathrm{DAS}$ & $52 \mathrm{DAS}$ \\
\hline $\mathrm{N} 2 \mathrm{~K} 0=\mathrm{a} \cdot \exp \left(-0.5((\mathrm{xb}) / \mathrm{c})^{\wedge} 2\right)$ & $105,567^{*}$ & $63,891^{*}$ & $12,037^{*}$ & 0,999 & $58 \mathrm{DAS}$ & $51 \mathrm{DAS}$ \\
\hline $\mathrm{L} 1=\mathrm{a} /(1+\exp (-(\mathrm{x}-\mathrm{b}) / \mathrm{c}))$ & $80,863^{*}$ & $49,302^{*}$ & $5,35^{*}$ & 0,999 & $70 \mathrm{DAS}$ & $49 \mathrm{DAS}$ \\
\hline $\mathrm{L} 2=\mathrm{a} /\left(1+(\mathrm{x} / \mathrm{b})^{\wedge} \mathrm{c}\right)$ & $127,826^{*}$ & $52,91^{*}$ & $-6,203^{*}$ & 0,997 & $70 \mathrm{DAS}$ & 49 DAS \\
\hline $\mathrm{L} 3=\mathrm{a} /(1+\exp (-(\mathrm{x}-\mathrm{b}) / \mathrm{c}))$ & $88,447^{*}$ & $48,383^{*}$ & $4,352^{\mathrm{ns}}$ & 0,992 & 70 DAS & $48 \mathrm{DAS}$ \\
\hline
\end{tabular}

(*): significativo (ns): não significativo a $5 \%$ pelo teste $\mathrm{T}$. 
Tabela 3. Equações que estimam o acúmulo de potássio na planta (CKPL), em kg ha ${ }^{-1}$, em função dos dias após semeadura (DAS), para diferentes doses de $\mathrm{N}$ e $\mathrm{K}$ e diferentes lâminas de irrigação.

\begin{tabular}{|c|c|c|c|c|c|c|}
\hline \multirow{3}{*}{ Modelos de CKPL } & \multirow{2}{*}{\multicolumn{3}{|c|}{ Parâmetros }} & \multirow[b]{3}{*}{$\mathrm{R}^{2}$} & \multicolumn{2}{|c|}{ Idade (DAS) } \\
\hline & & & & & $\begin{array}{c}\text { Para } \\
\text { CKPL }\end{array}$ & $\begin{array}{c}\text { Para taxa } \\
\text { de }\end{array}$ \\
\hline & A & b & c & & máximo & máximo \\
\hline $\mathrm{N} 1 \mathrm{~K} 1=\mathrm{a} \cdot \exp \left(-0.5((\mathrm{x}-\mathrm{b}) / \mathrm{c})^{\wedge} 2\right)$ & $438,746^{*}$ & $80,343^{*}$ & $\begin{array}{c}17,82^{*} \\
-\end{array}$ & 0,996 & $70 \mathrm{DAS}$ & $62 \mathrm{DAS}$ \\
\hline $\mathrm{N} 2 \mathrm{~K} 2=\mathrm{a} /\left(1+(\mathrm{x} / \mathrm{b})^{\wedge} \mathrm{c}\right)$ & $520,122^{*}$ & $59,995^{*}$ & $\begin{array}{c}7,095^{*} \\
-\end{array}$ & 0,999 & 70 DAS & $57 \mathrm{DAS}$ \\
\hline $\mathrm{N} 3 \mathrm{~K} 3=\mathrm{a} /\left(1+(\mathrm{x} / \mathrm{b})^{\wedge} \mathrm{c}\right)$ & $417,135^{*}$ & $60,101^{*}$ & $5,613^{*}$ & 0,991 & 70 DAS & $56 \mathrm{DAS}$ \\
\hline $\mathrm{N} 0 \mathrm{~K} 2=\mathrm{a} /(1+\exp (-(\mathrm{x}-\mathrm{b}) / \mathrm{c}))$ & $246,369^{*}$ & $56,11^{*}$ & $6,283^{*}$ & 0,999 & $70 \mathrm{DAS}$ & $56 \mathrm{DAS}$ \\
\hline $\mathrm{N} 2 \mathrm{~K} 0=\mathrm{a} /(1+\exp (-(\mathrm{x}-\mathrm{b}) / \mathrm{c}))$ & $213,378^{*}$ & $52,359^{*}$ & $5,562^{*}$ & 0,999 & $70 \mathrm{DAS}$ & $52 \mathrm{DAS}$ \\
\hline $\mathrm{L} 1=\mathrm{a} \cdot \exp \left(-0.5((\mathrm{x}-\mathrm{b}) / \mathrm{c})^{\wedge} 2\right)$ & $262,078^{*}$ & $71,889^{*}$ & $14,15^{*}$ & 0,999 & 70 DAS & $57 \mathrm{DAS}$ \\
\hline $\mathrm{L} 2=\mathrm{a} /(1+\exp (-(\mathrm{x}-\mathrm{b}) / \mathrm{c}))$ & $525,472^{*}$ & $63,16^{*}$ & $9,233^{\mathrm{ns}}$ & 0,992 & $70 \mathrm{DAS}$ & $63 \mathrm{DAS}$ \\
\hline $\mathrm{L} 3=\mathrm{a} /(1+\exp (-(\mathrm{x}-\mathrm{b}) / \mathrm{c}))$ & $286,712^{*}$ & $53,739^{*}$ & $5,473^{*}$ & 0,999 & 70 DAS & $53 \mathrm{DAS}$ \\
\hline
\end{tabular}

(*): significativo (ns): não significativo a $5 \%$ pelo teste $\mathrm{T}$.

\section{CONCLUSÕES}

Doses superiores a 140 e $260 \mathrm{~kg} \mathrm{ha}^{-1}$ de $\mathrm{N}$ e $\mathrm{K}$, respectivamente, não promoveram aumento no acúmulo de fitomassa seca total.

As doses crescentes de nitrogênio garantiram os maiores acúmulos deste nutriente ao longo do ciclo da planta e as doses de $\mathrm{N}$ de 91 e de $140 \mathrm{~kg} \mathrm{ha}^{-1}$, e de $\mathrm{K}$ de 174 e de $260 \mathrm{~kg}$ ha ${ }^{1}$, e a lâmina de $349 \mathrm{~mm}$ proporcionaram maior acúmulo de potássio na planta.

\section{REFERÊNCIAS BIBLIOGRÁFICAS}

ALLEN, R. G. et al. Crop evapotranspiration: guidelines for computing crop water requirements. Rome: FAO, 1998. 297 p.

BAR-YOSEF, B. Advances in fertigation. Advances in agronomy, Delaware, v. 65, p. 177, 1999.

BENINCASA, M. M. P. Análise de crescimento de plantas: noções básicas. 2. ed. Jaboticabal: FUNEP, 2003. 41 p.

EMPRESA BRASILEIRA DE PESQUISA AGROPECUÁRIA. Manual de métodos de análise de solos. Rio de Janeiro, 1997. 212 p.

EMPRESA BRASILEIRA DE PESQUISA AGROPECUÁRIA. Sistema brasileiro de classificação de solos. Brasília, DF: EMBRAPA, 1999. 412 p. 
FARIAS C. H. A. et al. Crescimento e desenvolvimento da cultura do melão sob diferentes lâminas de irrigação e salinidade da água. Revista Brasileira de Engenharia Agrícola e Ambiental, Campina Grande, v. 7, n. 3, p. 445-450, 2003.

LIMA, A. A. Absorção e eficiência de utilização de nutrientes por híbridos de melão (Cucumis melo L.). 2001. 60 f. Dissertação (Mestrado em Agronomia) Universidade Federal do Ceará, Fortaleza, 2001.

OLIVEIRA, F. A. et al. Acúmulo e partição de matéria seca, nitrogênio e potássio pelo meloeiro fertirrigado. Bioscience Journal, Uberlândia, v. 25, n. 3, p. 24-31, 2009.

RIBEIRO JÚNIOR, J. I. Análises estatísticas no SAEG. Viçosa: UFV, 2001. 301 p.

SILVA JÚNIOR, M. J. Acúmulo de matéria seca e absorção de nutrientes pelo meloeiro pele de sapo. Revista Brasileira de Engenharia Agrícola e Ambiental, Campina Grande, v. 10, n. 2, p. 364-368, 2006.

SILVA, M. C. de C. Crescimento, produtividade e qualidade de frutos do meloeiro sob diferentes níveis de salinidade da água de irrigação e cobertura do solo. $2001.65 \mathrm{f}$. Dissertação (Mestrado em Agronomia) Escola Superior de Agricultura de Mossoró, Mossoró, 2002.

SOUSA, V. D. de; COELHO, E. F. Manejo de fertirrigação em fruteiras. In: FOLEGATTI, M. V (Ed.). Fertirrigação: flores, frutas e hortaliças. Guaíba: Agropecuária 2, 2001. p. 289317.

TAIZ, L.; ZEIGER, E. Fisiologia vegetal. 3. ed. Porto Alegre: Artmed, 2004. 719 p.

TEDESCO, M. J. et al. Análise de solo, plantas e outros materiais. Porto Alegre: UFRGS, 1995. $174 \mathrm{p}$.

VIVANCOS, A. D. Fertirrigacion. Madri: Mundi-Prensa, 1996. 233 p. 